\title{
First attempts to obtain a reference drift curve for traditional olive grove's plantations following ISO 22866
}

\author{
Emilio Gil ${ }^{\mathrm{a}, *}$, Jordi Llorens ${ }^{\mathrm{b}}$, Montserrat Gallart ${ }^{\mathrm{a}}$, Jesús A. Gil-Ribes ${ }^{\mathrm{b}}$, Antonio Miranda-Fuentes ${ }^{\mathrm{b}}$ \\ a Department of Agri Food Engineering and Biotechnology, Universitat Politècnica de Catalunya, Esteve Terradas 8, Campus del Baix Llobregat D4, 08860 Castelldefels, \\ Barcelona, Spain \\ b Dpto. Ingeniería Rural, Área de Mecanización y Tecnología Rural, Universidad de Córdoba, 14005 Córdoba, Spain
}

\section{A R T I C L E I N F O}

Article history:

Received 4 October 2017

Received in revised form 23 January 2018

Accepted 23 January 2018

Available online $\mathrm{xxx}$

Editor: D. Barcelo

Keywords:

Olive tree

Deposition

Wind speed

ISO

Airborne deposition
Spray drift

\section{A B S T R A C T}

The current standard for the field measurements of spray drift (ISO 22866) is the only official standard for drift measurements in field conditions for all type of crops, including bushes and trees. A series of field trials following all the requirements established in the standard were arranged in a traditional olive grove in Córdoba (south of Spain). The aims of the study were to evaluate the applicability of the current standard procedure to the particular conditions of traditional olive trees plantations, to evaluate the critical requirements for performing the tests and to obtain a specific drift curve for such as important and specific crop as olive trees in traditional plantations, considering the enormous area covered by this type of crop all around the world. Results showed that the field trials incur a very complex process due to the particular conditions of the crop and the very precise environmental requirements. Furthermore, the trials offered a very low level of repeatability as the drift values varied significantly from one spray application to the next, with the obtained results being closely related to the wind speed, even when considering the standard minimum value of $1 \mathrm{~m} \cdot \mathrm{s}^{-1}$. The collector's placement with respect to the position of the isolated trees was determined as being critical since this substantially modifies the ground deposit in the first $5 \mathrm{~m}$. Even though, a new drift curve for olive trees in traditional plantation has been defined, giving an interesting tool for regulatory aspects. Conclusions indicated that a deep review of the official standard is needed to allow its application to the most relevant orchard/fruit crops.

(C) 2017 .

\section{Introduction}

The legal framework concerning the use of pesticides in Europe changed dramatically after the official publication of the Sustainable Use of Pesticides (Parliament, 2009a). However, in addition, the publication of European Directive 2009/127/EC (Parliament, 2009b), amending Directive 2006/42/EC with regard to the machinery used for pesticide application, which regulates the environmental aspects of pesticide application equipment newly manufactured in Europe, and the Plant Protection Products Regulation 1107/2009 (Parliament, 2009c) has recently generated substantial changes in crop protection activities and has forced all the stakeholders to undertake considerable efforts to implement these mandatory rules.

One of the aspects that has undergone changes is related to the required spray application conditions necessary for a particular active substance, including its safe use, thus avoiding both environmental risks and pollution. This issue is directly related to the establishment of so-called buffer zones, whereby a pesticide non-use zone shall be

\footnotetext{
* Corresponding author.

Email addresses: emilio.Gil@upc.edu (E. Gil); jordi.llorens@eagrof.udl.cat (J. Llorens); montserrat.gallart@upc.edu (M. Gallart); gilribes@uco.es (J.A. Gil-Ribes); g62mifua@uco.es (A. Miranda-Fuentes)
}

implemented in those situations where the use of plant protection products could incur a risk for the environment (Burn, 2003; de Snoo and de Wit, 1998). This fact has led to the derivation of a wide range of solutions among EU members, where the relative importance afforded has depended on aspects such as the degree of hazard of the active ingredient or the technological level of the equipment used and its ability to reduce drift.

The technological level of the equipment for crop protection, together with the conditions of use therefore and the criteria for the selection of operating parameters have a direct influence on the risk of contamination. Several improvements to quantify these effects have been carried out in the past (Doruchowski et al., 2014; Doruchowski et al., 2013; Wang and Rautmann, 2008), and some research efforts have been applied to establish classification schemes based on drift values that have been measured using different techniques or procedures (De Schampheleire et al., 2008; Herbst, 2001; van De Zande et al., 2008; van de Zande et al., 2015). Nevertheless, there are still important aspects concerning drift measurement that require further improvement, especially regarding the application of pesticide in the likes of orchards, vineyards, and citrus or olive groves).

Currently, three official procedures are recognised for drift measurements: ISO 22856: 2008, the standard protocol for evaluating the drift from nozzles in a wind tunnel under controlled conditions in the laboratory (ISO 22856, 2008); ISO 22866:2005 for drift measure- 
ments under field conditions, for both field crop and orchard sprayers (ISO 22866, 2005); and the latest developed ISO 22401:2015 for the measurement of the drift potential of boom sprayers (ISO 22401, 2015). A close look to these standards reveals that different aspects, parameters, and procedures must be considered for each procedure, leading in some cases to difficulties with comparing the obtained results (Balsari et al., 2007; van de Zande et al., 2000). It is also important to note that only ISO 22866 is fully dedicated to field trials, being the only standard capable of evaluating the pesticide application equipment under real-world conditions. This fact is especially important in the case of field trials to determine drift measurements when using orchard sprayers for bush and tree crops. Even if this standard and the results obtained after its application (Ganzelmeier et al., 1995) are used as a reference for the official pesticide registration process (FOCUS, 2001, 2014; Rautmann et al., 2001) it is clear that external factors such as weather conditions, the canopy structure, and dimensions and distribution of the trees in the field have a great effect on the measured values. Additionally, it is worth noting that the complete application of this standard would lead to some great difficulties and would incur a considerable use of time and labour (De Schampheleire et al., 2008; Ravier et al., 2005; Rimmer et al., 2009). Furthermore, the difficulty with obtaining good repeatability of the results has also been demonstrated (Balsari et al., 2005; Ozkan, 1998; van de Zande et al., 2000). Great variability due to the influence of environmental conditions is observed, resulting in an extremely time consuming, complex, and difficult process. Other researchers (Arvidsson et al., 2011) concluded that a sequence of experiments could require several hours, even if the line of the measurements did not have to be changed as long as the average wind deviation was in the range of $\pm 30^{\circ}$ from the original line. Furthermore, it is necessary to consider the specific requirements regarding the deposition values at different measurement points both in the horizontal and vertical collectors, which gives rise to major difficulties when attempting to completely attain the requirements.

Considering the fact that ISO 22866: 2005 is the only official procedure for measuring the drift generated by orchard sprayers in a field, and considering the great difficulties associated with its application, as determined by previous research efforts, this study set out to evaluate the adaptability of the standard for drift measurement to traditional olive groves and to propose a specific drift curve for this kind of crop to be used for regulatory aspects, given that olives are one of the most popular and widespread crops in the southern part of Europe. A series of field trials that precisely follow the standard requirements were arranged in traditional olive groves in the south of Spain. The specific objectives of this research were: 1) to evaluate the effect of each requirement on the final results, 2) to quantify the difficulties related to satisfying all the very restrictive requirements and 3) to propose and olive-drift curve and compare it with the actual most close available (drift curve in late growth stage fruit crops and hops).

\section{Materials and methods}

\subsection{Specifications of ISO 22866:2005}

\subsubsection{Main changes related to hedgerow crops}

As mentioned above, ISO 22866:2005 was mainly devised for to be applied for field crop drift measurements (Tavares et al., 2017), even if several attempts for drift measurements in orchard/vineyard sprayers have been developed in the last years (García-Ramos et al., 2015; Grella et al., 2017) however, its application to the case of large, isolated and irregular trees, such as traditional olive trees plantations, represents additional difficulties and considerable requirements for its adaptation. In this research, a specific protocol was developed such that all of the requirements set out in the standard were satisfied while including the specific characteristics of the crop. The main change was related to the number of samples: The total number of samples at each distance should be, according to the standard's requirements, such that a confidence interval of $95 \%$ can be achieved for the mean deposit at a point $5 \mathrm{~m}$ from the edge of the directly sprayed area. Nevertheless, irregularities in the shapes of the olive tree crowns made necessary to increase the number of replications of the whole test. Furthermore, it was necessary to adapt the sampling area according to the distance between the trees (Fig. 1).

\subsubsection{Experimental design}

The tests were carried out by placing horizontal collectors (Petri dishes) on the ground, and vertical collectors (drift masts using nylon line). Six replications of the test were performed.

\subsubsection{Horizontal collectors}

Petri dishes were used as horizontal collectors to collect the drift being deposited on the ground. The sampling area was placed starting on the bare ground at $3 \mathrm{~m}$ distance of the outer tree row and the dishes were set at points between $1 \mathrm{~m}$ and $20 \mathrm{~m}$ from the beginning of the sampling area, following the standard requirements. Thus, from 1 to $5 \mathrm{~m}$, there was $1 \mathrm{~m}$ distance between the collectors, but this was increased to $2.5 \mathrm{~m}$ between 5 and $10 \mathrm{~m}$ from the trees, and to $5 \mathrm{~m}$ between 10 and $20 \mathrm{~m}$. A total of six lines were established, starting from the centre of the first tree's trunk (Figs. 1 and 2a). Petri dishes were $150 \mathrm{~mm}$ in diameter, so a total of $1060.3 \mathrm{~cm}^{2}$ of sampling surface was established over all the sampling distances (the standard establishes a minimum of $1000 \mathrm{~cm}^{2}$ ).

\subsubsection{Vertical collectors}

Two 6-m vertical masts, especially designed for drift quantification tests (Summaprecisió S.L., Lleida, Spain) (Gregorio et al., 2015) were placed at points $5 \mathrm{~m}$ and $10 \mathrm{~m}$ from the outermost last tree row (Figs. 1 and 2b). Each mast supported two continue nylon collector's lines used with the purpose to determine the vertical distribution of the spray drift. Every one of the nylon collector line had a length of $6 \mathrm{~m}$ and a diameter of $3 \mathrm{~mm}$.

\subsection{Field selection and specific technical requirements for weather condition's record}

\subsubsection{Characteristics of the selected field}

A previous selection of potential candidate parcels where the drift test could be performed was made in two commercial olive farms with traditional planting patterns, located in the province of Córdoba (Southern Spain). These were selected from a large area evaluated using aerial views obtained from Google Earth (Version 5.1.3533.1731, Mountain View, CA). The selected fields featured well established trees of the Picual and Hojiblanca varieties. The planting pattern had a tree and row spacing of $12 \mathrm{~m}$ in a 'quincunx' scheme. From the selected farms, a total of 18 fields (Fig. 3) were chosen for satisfying the requirements of the ISO 22866, i.e., for having a predominant wind direction corresponding with the perpendicular one to the trees rows in the field, accomplishing at the same time the presence of a bare space of at least $40 \mathrm{~m}$ next to their most external row of trees, as well as crop lines that were parallel to the field edges. Moreover, a detailed study of the weather conditions was undertaken based on historical data recorded by nearby weather stations from the Agroclimatic Information Network of Andalusia (Junta de Andalucía, 2015a, b, c) of the target zone. 


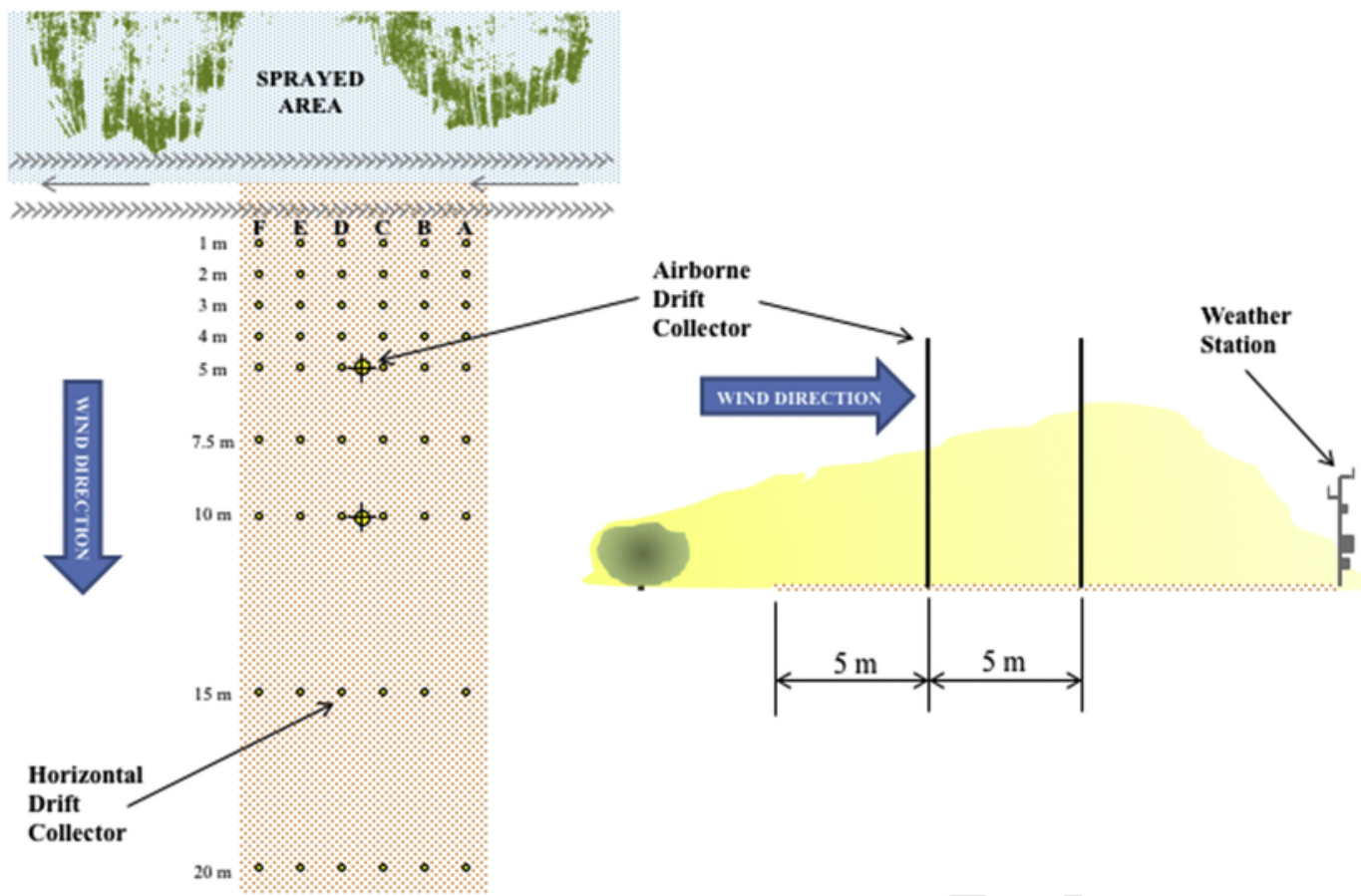

Fig. 1. Experimental setup with a digital representation of the closest trees.
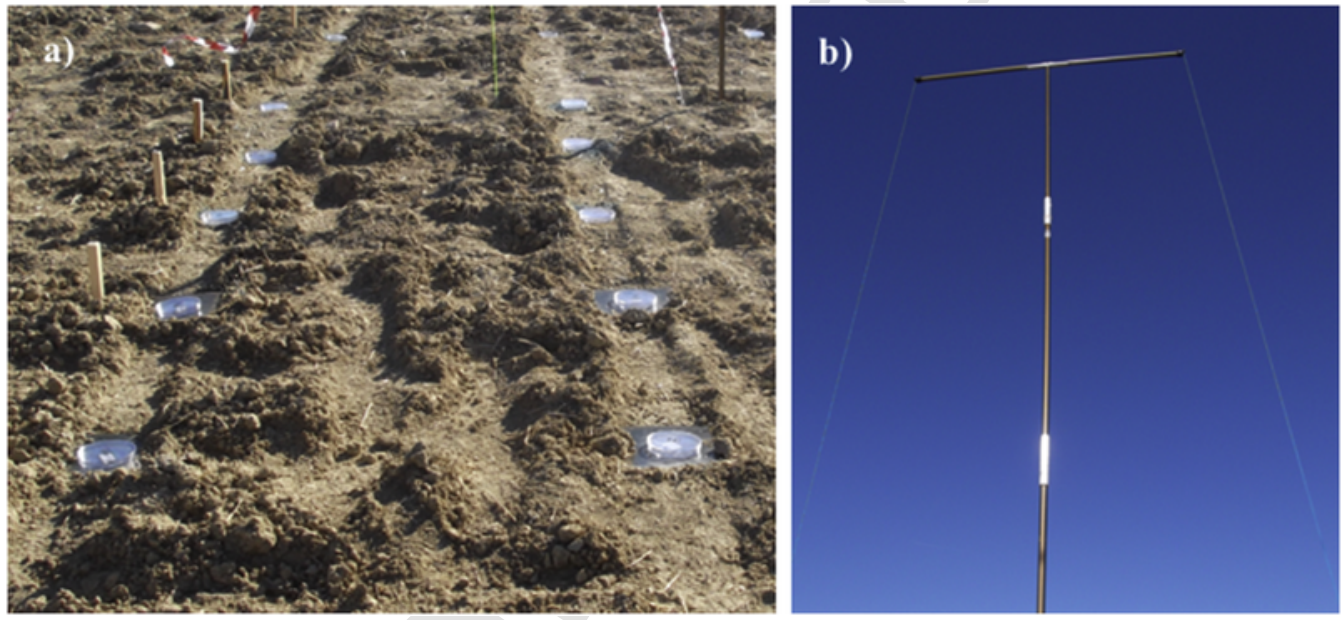

Fig. 2. a) Petri dishes disposed as horizontal collectors; and b) vertical mast with nylon collectors.

\subsubsection{Previous evaluation of meteorological conditions}

In order to determine the suitability of the orientation of each one of the selected fields, a complete wind speed/direction monitoring was performed. A dedicated weather station (CR800, Campbell Scientific Inc., Logan, UT, USA) was used to monitor the wind speed and direction, as well as the temperature and relative humidity of the pre-selected fields. A 2D ultrasonic anemometer WindSonic 232 (Campbell Scientific Inc.), able to measure the wind speed and direction, has a measurement range of up to $60 \mathrm{~ms}^{-1}$, with a resolution of $0.01 \mathrm{~ms}^{-1}$ and $1^{\circ}$ for the wind direction measurement. The sensor presents up to four outputs per second when connected using an RS-232 communication port, although this study collected only one output per second. Measurements of the temperature and the relative humidity were performed using a CS215 temperature and relative humidity probe (Campbell Scientific Inc.), with a measurement range from 0 to $100 \%$ relative humidity and a temperature of -40 to $+70^{\circ} \mathrm{C}$. Two probes placed at different heights made possible to calculate the temperature difference. Special control software was programmed into the application PC200W Datalogger Starter Software ${ }^{\circledR}$ (Campbell Scientific Inc.) to perform the measurements and the process of data transfer. The measurements were taken with a frequency of $0.1 \mathrm{~Hz}$, stored in the data logger's internal memory and monitored real-time using a laptop computer. Data monitoring was performed for a total of 12 days, divided into two periods of 3 and 9 days.

\subsubsection{In-field observations}

In addition to the aerial pictures, it was necessary to actually visit the fields to check for possible circumstances that could limit or even prevent their use in the study, such as the presence of an excessive 

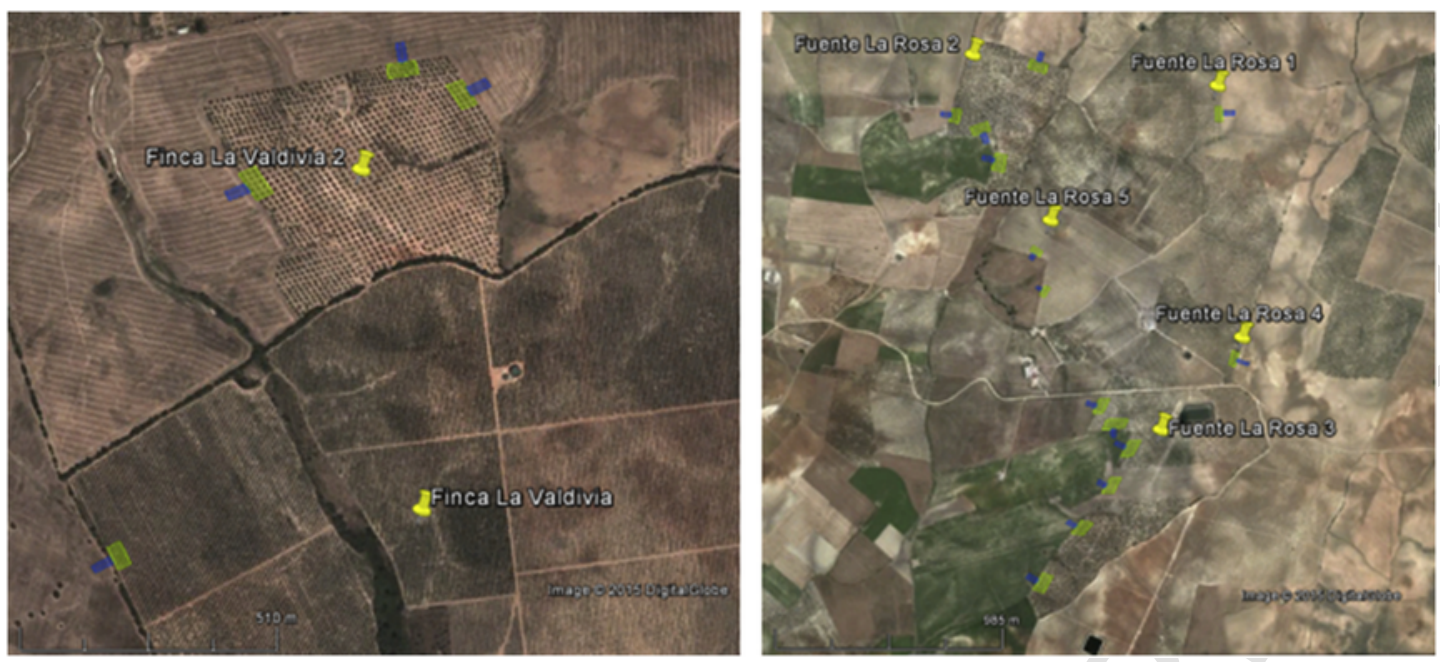

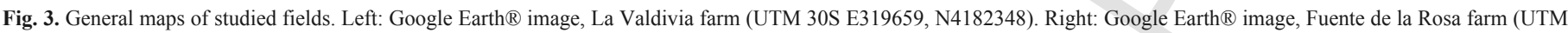
30S E340364, N4176028).

slope, gullies, small streams, level differences between adjacent fields, etc. Finally, taking all of the aforementioned criteria into account, one field (UTM 30 S E340178 m, N4175345 m) was selected as the most suitable for conducting the trial.

\subsection{Characterisation of vegetation (canopy dimensions)}

Due to the size and irregularity of traditional olive trees, an exhaustive characterisation of all the trees involved in the trial was performed. This was a key aspect of the trial, as it was planned to have a representative sampling area that ranged from full vegetation (at the centre of the trees) to none at all (gaps between trees). A total of 15 trees from three different rows were characterised both manually and electronically using a LiDAR scanner.

\subsubsection{Manual characterisation of the canopy}

A manual characterisation of the randomly selected trees was performed according to the methodologies described in Miranda-Fuentes et al. (2015). Thus, the ellipsoid volume $\left(V_{E}\right)$, the projected area $\left(A_{P A}\right)$ and the mean vector $(M V)$ were considered. The ellipsoid method consists of measuring two tree crown diameters along with the crown height, and calculating the volume of a theoretical ellipsoid $\left(V_{E}\right)$ that presents these dimensions. The projected area method consists of determining the area projected on the ground by the tree crown $\left(A_{P A}\right)$ by taking the coordinates of eight projected points. Last, the Mean Vector method consists of measuring the distance from the centre of the tree crown to eight projected peripheral points around it, and calculating the mean value $(M V)$. The three parameters described proved to be accurate in the traditional cultivation system.

\subsubsection{Electronic canopy measurements using LiDAR sensor}

A complete electronic characterisation of the trees was performed with a 2D terrestrial LiDAR (LMS-111, Sick, Dusseldorf, Germany). This laser scanner has a resolution up to $0.25^{\circ}$, with a scanning angle of $270^{\circ}$ in a range up to $20 \mathrm{~m}$. The laser scanner was mounted on a specially-designed platform attached to the rear part of the tractor, such that it was positioned $1.7 \mathrm{~m}$ above the ground. All the selected trees were characterised by driving the tractor between two rows at a constant speed of $1 \mathrm{~km} \mathrm{~h}^{-1}$. An AGGPS162 GPS device (Trimble Navigation Ltd., Sunnyvale, CA, USA) with EGNOS differential correction was used to correlate the data obtained from both sides of the trees, and different objects of known dimensions were used as fixed references as done in other studies (Llorens et al., 2011). Data was represented and delimited with CloudCompare software (Girardeau-Montaut, 2011) and analysed using a particular code developed using the $\mathrm{R}$ software (R Core Team, 2012). The tree volume of every single tree was obtained by dividing the entire point cloud into horizontal slices measuring $0.1 \mathrm{~m}$ high, as described by $\mathrm{Xu}$ et al. (2013).

\subsection{Materials}

\subsubsection{Sprayer and spray parameters during the spraying process}

All the treatments were executed using a $2000 \mathrm{~L}$ commercial air blast sprayer with an axial fan (Osuna-Sevillano, Jauja, Spain) equipped with hollow cone nozzles (Albuz, Saint-Gobain Ceramiques Avancees Desmarquest, Evreux, France). Prior to the application, the sprayer was calibrated to ensure that the applied volumes were in accordance with the expected ones. The spray parameters are listed in Table 1 . The spray volume of $1000 \mathrm{~L} \mathrm{ha}^{-1}$ was chosen as this is typical of the volume applied on the zone. Forward speed of $3 \mathrm{~km} \cdot \mathrm{h}^{-1}$ was adopted and maximum work pressure of 15 bar was not fixed. As the main objective of this research was to evaluate the adaptability of the drift standard and to develop an alternative drift curve for traditional olive trees plantations, all the trials were executed using the same sprayer maintaining in all cases the same working parameters. Selection of both, type of sprayer and working parameters, was made according the most popular and widely used for olive trees.

Table 1

Operational parameters of the trial.

\begin{tabular}{ll}
\hline Parameter & Value \\
\hline Nozzle model and colour & Albuz ATR Blue \\
Number of open nozzles & $14(2 \times 7)$ \\
Pressure (bar) & 10.0 \\
Liquid flow rate $\left(1 \cdot \mathrm{min}^{-1}\right)$ & 47.88 \\
Spray volume $\left(1 \cdot \mathrm{ha}^{-1}\right)$ & 1008 \\
Forward speed $\left(\mathrm{km} \cdot \mathrm{h}^{-1}\right)$ & 2.85 \\
VMD $(\mu \mathrm{m})$ & 97 \\
PTO speed $(\mathrm{rpm})$ & 467 \\
Air flow rate $\left(\mathrm{m}^{3} \cdot \mathrm{s}^{-1}\right)$ & 9.40 \\
\hline
\end{tabular}

VMD (volumetric mean diameter) and PTO (power take-off). 


\subsubsection{Spray tracer}

The spray tracer used in the trial was E102 Tartrazine food dye. This was selected as it exhibits a very low degree of degradation (Pergher, 2001) and toxicity. The large number of repetitions and the high applied volumes made it necessary to use an innocuous tracer so as not to harm either the environment or the operator. Furthermore, previous trials showed that Tartrazine deposits could be detected even at very low levels, due to the high concentration of the commercial product $(80 \%)$ and the easiness to be quantified by spectrophotometry techniques.

\subsection{Test performance}

A team of seven operators performed the trials over a period of one week. Before the trials, the four lines of ground collectors closest to the sprayer were fixed to wooden surfaces by adhesive tape in order to prevent them from being disturbed by the air flow generated by the orchard sprayer. One operator continuously checked the wind speed and direction to determine those periods within the ranges established by the standard. The rule to be observed was that the trial could begin once the favourable conditions had been maintained for $1 \mathrm{~min}$ to avoid intervals that would make the replication invalid. Once the conditions were deemed favourable, i.e., the weather parameters presented values inside the ranges set by the standard, a sample of the spray mix from the sprayer tank was collected into a plastic tube to determine the exact concentration of the spray tracer. Simultaneously, the remaining operators removed the ground collectors' covers, and finally the spraying process started.

The sprayer would then treat three complete tree rows, spraying a total area of $120 \mathrm{~m}^{2}$, i.e., $50 \mathrm{~m}$ long by 3 rows of $10 \mathrm{~m}$ wide each, following the track shown in Fig. 4. The weather conditions data recorded by the weather station during the trial were properly stored. Once the spraying process was completed, the collectors' covers were immediately replaced and labelled, after which another liquid sample was collected from the sprayer tank. Then, all of the Petri dishes were stored out of the light and the nylon collectors were cut $(1 \mathrm{~m}$ length each one) and classified into six positions corresponding the different heights of the mast. Nylon samples were packaged into individual plastic bags and stored immediately in dark conditions. For the collection process, latex gloves were used and continuously replaced in order to prevent undesirable contamination of the samples.

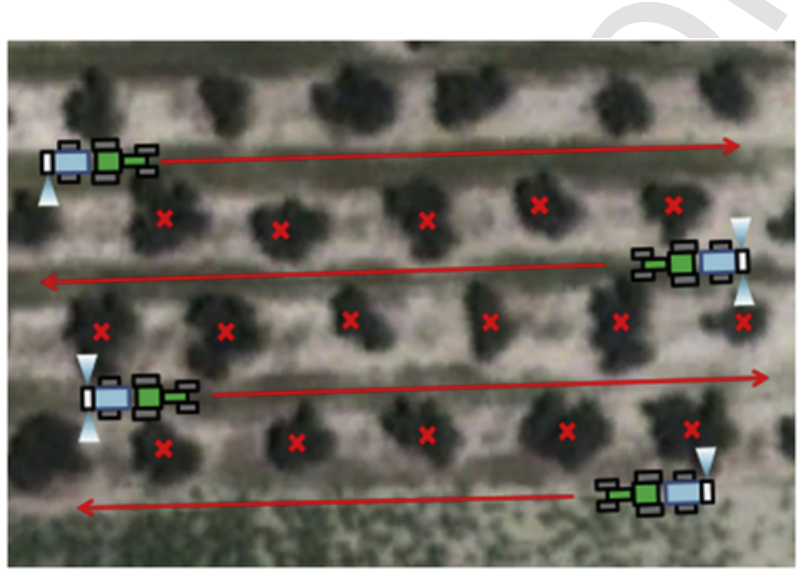

Fig. 4. Sprayer track during the trial.

\subsection{Laboratory analysis}

\subsubsection{Spectrophotometer calibration}

To analyse the tracer concentration, the absorbance was recorded using a spectrophotometer (Synergy HTX, BioTek Instruments, Inc., Winooski, VT, USA) set to a wavelength of $427 \mathrm{~nm}$, and capable of detecting a tracer concentration of between $0.1 \mathrm{ppm}$ and $150 \mathrm{ppm}$.

\subsubsection{Collector's washing process and deposit quantification}

For every single soil sample, $100 \mathrm{~mL}$ of distilled water were added to the dishes, which were then shaken for $5 \mathrm{~min}$. The washing solution was then placed in a $150-\mathrm{mL}$ glass flask and six samples were extracted with an automatic pipette and deposited into a 96-well plate. Each plate had eight blank wells containing deionised water to calibrate the zero value on the device. The same procedure was followed for the aerial samples obtained from the vertical masts, adding $100 \mathrm{~mL}$ of distilled water to every plastic container containing the nylon sample.

\subsubsection{Determination of tracer deposit}

Results were obtained in terms of surface deposit $\left(\mu \mathrm{L} . \mathrm{cm}^{-2}\right)$ and spray deposit percentage (\%). The first step was to obtain the total amount of spray deposit per unit area, as defined by Eq. (1).

$$
\beta_{d e p}=\frac{\left(A b s_{\text {samp }}-A b s_{b l k}\right) \times V_{d i l} \times 10^{6}}{A b s_{\text {spray }} \times A_{c o l}}
$$

where, $\beta_{\text {dep }}$ is the deposit per unit area $\left(\mu \mathrm{L} \cdot \mathrm{cm}^{-2}\right), A b s_{\text {sampl }}$ is the fluorimeter absorbance value of the sample, $A b s_{b l k}$ is the fluorimeter absorbance reading of the blanks, $V_{d i l}$ is the volume of the dilution liquid used to solute the tracer from the collector, expressed in litres (L), $A b s_{\text {spray }}$ is the fluorimeter absorbance value of the tank spray sample, and $A_{c a l}$ is the projected area of the collector for catching the spray drift, expressed in $\mathrm{cm}^{2}$.

The spray deposit percentage was calculated using Eq. (2).

$\beta_{d e p \%}=\frac{\beta_{d e p} \times 10000}{\beta_{V}}$

where, $\beta_{d e p \%}$ is the spray deposition percentage value (\%) and $\beta_{V}$ is the applied spray volume, expressed in $\mathrm{L} \cdot \mathrm{ha}^{-1}$.

\subsubsection{Evaluation of the accomplishment of the standard requirements \\ Given the standard requirements, it is mandatory to accomplish} specific and restrictive weather conditions (Table 2). Once all the values about deposit per unit area were calculated, ISO 22866 requirements were checked in order to determine whether the results of the test were valid. These standard requirements differ considerably between the ground and airborne samples. In the first case, it is necessary to ensure that the number of samples at each sampling distance is sufficient to produce a representative result. This requirement is regarded as having been satisfied when a confidence interval of $95 \%$ can be achieved for the mean deposit at a distance of $5 \mathrm{~m}$ from the edge of the directly-sprayed area (Section 3.6 of the standard). In the case of the airborne spray drift collector, the total height must be sufficient to collect at least the $90 \%$ of the total spray drift, with the 
Table 2

Summary of the weather conditions for the six replications of the drift trials.

\begin{tabular}{|c|c|c|c|c|c|c|c|c|}
\hline Weather parameters & & ISO 22866 limitation & Rep 1 & Rep 2 & Rep 3 & Rep 4 & Rep 5 & Rep 6 \\
\hline Date test & & & $26 / 01 / 2015$ & $27 / 01 / 2015$ & $27 / 01 / 2015$ & $28 / 01 / 2015$ & $28 / 01 / 2015$ & $28 / 01 / 2015$ \\
\hline Start time (hh:mm:ss) & & & $16: 10: 00$ & $16: 42: 00$ & $17: 28: 00$ & $12: 12: 36$ & $14: 19: 00$ & $14: 49: 00$ \\
\hline End time (hh:mm:ss) & & & $16: 15: 58$ & $16: 47: 58$ & $17: 33: 00$ & $12: 19: 10$ & $14: 23: 58$ & $14: 53: 58$ \\
\hline Temperature height 1 & $\mathrm{~T}_{1}$ & 5 to $35^{\circ}$ & 15.78 & 16.39 & 16.45 & 10.72 & 14.46 & 15.4 \\
\hline Humidity height 1 & $\mathrm{HR}_{1}$ & No limitation & 32.34 & 40.82 & 41.57 & 64.46 & 49.98 & 46.63 \\
\hline Temperature height 2 & $\mathrm{~T}_{2}$ & 5 to $35^{\circ}$ & 15.56 & 16.17 & 16.33 & 10.45 & 14.19 & 15.15 \\
\hline Humidity height 2 & $\mathrm{HR}_{2}$ & No limitation & 32.64 & 41.33 & 41.63 & 65.45 & 50.69 & 47.37 \\
\hline Mean wind speed & $\mathrm{WS}^{2}$ & $>1 \mathrm{~m} / \mathrm{s}$ & 1.06 & 1.45 & 1.29 & 2.28 & 1.57 & 1.38 \\
\hline Mean wind direction & WD & $0 \pm 30^{\circ}$ & 50.5 & 19.55 & 24.9 & 29.7 & 37.67 & 57.95 \\
\hline Wind outlier $(<1 \mathrm{~m} / \mathrm{s})$ & $\mathrm{WS}_{\text {OUT }}$ & $<10 \%$ & 48.02 & 8.8 & 17.8 & 2.52 & 19.3 & 22.6 \\
\hline Wind centered $\left(<45^{\circ}\right)$ & $\mathrm{WC}_{<45^{\circ}}$ & $>30 \%$ & 45 & 95 & 95 & 82 & 71 & 22 \\
\hline Wind not centered $\left(>45^{\circ}\right)$ & $\mathrm{WNC}_{>45^{\circ}}$ & $<70 \%$ & 55 & 5 & 5 & 16 & 29 & 78 \\
\hline
\end{tabular}

highest sample being compared with the others (Section 3.5 of the standard).

\subsubsection{Statistical analysis of the samples}

To numerically and statistically compare the different replications of the executed trials, a logarithmic transformation of the data was performed (Eq. (4)). This process was similar to that applied graphically by Ganzelmeier et al. (1995). The drift curve function is defined by Eq. (3):

$$
\beta_{d e p \%}=\mathrm{f}(d)+\mathrm{k}
$$

The linearized drift curve function using logarithm is defined by Eq. (4):

$$
\operatorname{Ln}\left(\beta_{d e p \%}\right)=(\operatorname{Ln} d)+\mathrm{k}
$$

where $\beta_{d e p \%}$ is the spray deposition percentage value (\%) (obtained from Eq. (2)), $d$ is the position of each collector expressed in $\mathrm{m}$, and $\mathrm{k}$ represents the effect of all the other factors that affect the results of the trial (sprayer, weather, ...).

These logarithm transformations (Eqs. (3) and (4)) enable us to obtain linear functions that are easily comparable through statistical tests. In this particular case, the analysis of covariance (ANCOVA) method was used (Poprawski et al., 1997; Serrano et al., 2000). The functions obtained for each replication were compared 2 by 2 in order to check the differences between the influence of the treatment categorical variable (replication) and the continuous co-variable (distance) on the measured dependent variable (deposit). Therefore, the interception of the factors replication*distance should be constant, assuming that there are no significant differences between each evaluated pair of replications. Prior to the analysis, the normality and homoscedasticity assumptions were checked. Residual analyses were also performed.

In addition, the drift functions were compared with the reference curves obtained in Rautmann et al. (2001) for FCLG (Fruit Crop Late Growth) and hops following a similar statistical procedure mentioned before. Similar comparison was made in Grella et al. (2017). All the statistical analysis was performed using R software (R Core Team, 2012).

\section{Results}

\subsection{Difficulties for a proper field selection}

The planning of the trials was difficult and it ultimately proved impossible to execute exactly as specified during the work week. The main problems and difficulties experienced during the trial are listed below.

\subsubsection{Tree and row spacing}

Traditional olive tree orchards present a very low tree density per area unit. This leads to a tree and row spacing that makes it difficult to perform a homogeneous spray application. Furthermore, plantation patterns are often irregular (Fig. 5), making the identification of tree rows difficult. Regarding drift quantification, the main problem arises from the rectangular sampling area that is defined by its length and width. Even if a very large area is assumed, the irregularities of the tree canopy cause even very low variations in the wind direction to produce very important differences in the deposition patterns. This prevents the repeatability of the trial and makes it much more difficult to accomplish the requirements of the standard, making it necessary to increase the number of replications of the trial.

\subsubsection{Structure of the property}

Traditional olive properties are generally quite small in the south of Spain (4.93 ha, Junta de Andalucía, 2015a) but are often located next to each other, thus forming extensive areas of trees. This makes it difficult to find suitable fields in which the trials can be performed due to the lack of empty space in which to place the sampling equip-

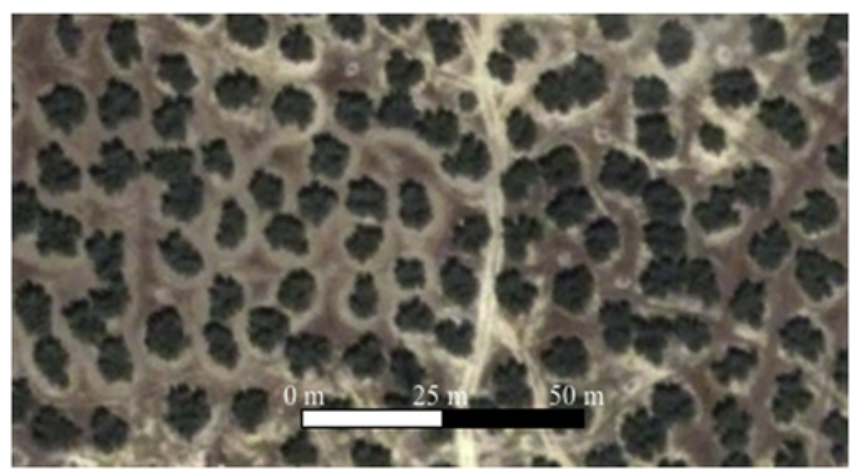

Fig. 5. Irregular plantation pattern in traditional olive grove in Martos (Jaén, Andalusia, Spain). 
ment. Even though this problem may not seem to be very important, in practice it is very salient, and thus greatly restricts the possibilities of field selection. The minimum dimensions of the sampling rectangle make it necessary to have a contiguous field that is not cultivated, with no valid spaces between fields, roads, or other bare spaces. Furthermore, there is another problem with the irregularity of the field borders in that they are not parallel with the tree rows. Therefore, the setup of the trial results impossible in these cases.

\subsubsection{Maximum average slope accepted}

Olive-growing areas are generally found on non-productive soils, near mountain ranges, due to the characteristics of the tree making it possible to take advantage of soils that are not suitable for arable or other fruit crops. As a result, it is typical for olive groves to have a very steep slope (usually between 10 and $20 \%$, or even more, Fig. 6). To quantify the airborne spray drift, masts containing airborne collectors must be placed in a horizontal field to guarantee a $\mathrm{n}$ efficient collection the spray drift at to each height. This requirement markedly limits the suitability of the fields.

\subsubsection{Row orientation according to prevailing wind direction}

Another issue that seriously limits the selection of a testing field is the orientation of the tree rows. The criterion required the selection of fields with a regular border perpendicular to the prevailing wind. This is another limiting factor, because most groves are oriented north-south, while the prevailing wind direction in this area is from the south-west $\left(220^{\circ}-230^{\circ}\right)$.

\subsection{Results of the trials}

\subsubsection{Weather conditions}

A summary of the recorded weather conditions for each replication is listed in Table 2. Only two of all of the intended replications could considered completely valid, according the standard requirements (see rep. 2 and rep. 4 on Table 2). It is noticeable that there are two issues that make most of the replications invalid, namely, the mean wind direction (WD) and the wind outlier $\left(\mathrm{WS}_{\mathrm{OUT}}\right)$, while the other requirements are, in this case, less restrictive. The wind direction ranged from $19.55^{\circ}$ to $57.95^{\circ}$.

Among the valid replications, the wind direction was marginal in Rep. 4, but slightly more centred in Rep. 2 . The wind speed values were $1.45 \mathrm{~m} \cdot \mathrm{s}^{-1}$ and $2.28 \mathrm{~m} \cdot \mathrm{s}^{-1}$, for Rep. 2 and Rep. 4, respectively. Considering the other replications of the trial, the wind speed values were found to all be between 1 and $2 \mathrm{~m} \cdot \mathrm{s}^{-1}$, with the exception of Rep. 4.
The different replications were done on different days, as listed in Table 2. On the first day, when the trial was set up, it was possible to perform only one replication. Two replications were done on the second day and three on the third. The duration of each replication, it ranged from $4^{\prime} 58^{\prime \prime}$ for replications 5 and 6 to $6^{\prime} 34^{\prime \prime}$ for replication 4 , with a mean value of $5^{\prime} 34^{\prime \prime}$. The time taken depended on the tractor driver, who had to get off the tractor to manually open/close the valves and select either a double- or single-side treatment, depending on the position of the tractor.

\subsubsection{Canopy characterisation}

The volume of the characterised trees was very high, as shown by the four characterisation methods (Table 3 ). The total height was in excess of $4.5 \mathrm{~m}$, with a minimum value of $4.10 \mathrm{~m}$ and a maximum of $5.00 \mathrm{~m}$. The height of the first leaf was $0.56 \mathrm{~m}$.

\subsubsection{Collected spray deposits}

The horizontal deposits collected by the Petri dishes are shown in Figs. 7 and 8. The data in the Figures correspond to results obtained according Eqs. (2) and (4), respectively.

The mean values of the drift curves were very similar, with the exception of Rep. 4 (Fig. 9). This is especially evident in Fig. 8, where the mean deposit for Rep. 4 is much higher than that obtained for the other replications. Even though differences in the distributions can be observed for different distances, the deposits at $20 \mathrm{~m}$ are greatly reduced in all cases. It is noticeable the presence of collected deposit percentages higher than $12 \%$ in punctual cases, like that found in Rep. 4. This circumstance could be caused by the fact that the treatment of subsequent tree rows presents such a low efficiency that significantly increase the amount of spray mix that is deposited in the collectors, out of the orchard. Therefore, the final situation found in these cases is similar to the one that would be expected after treating more than one time the same ground area.

Focusing on the variability of the deposits, the highest was obtained in Rep. 4 (Fig. 7), while the others behaved differently, with a higher variability appearing in Reps. 2 and 3 than in Reps. 1, 5, and 6. The logarithm functions for each replication are listed in Table 4, together with their coefficients of determination and their $P$-values.

Fig. 8 shows the ANCOVA results for Reps. 2 and 4, that is, the two valid replications of the trial. The treatment also has an effect, as the factor replication was shown to have a very low $P$-value. Furthermore, the interaction between the distance and replication factors, the slope, has a significant influence, while the obtained P-value is $<0.05$. This fact means that there is a different decrease in the collected deposits in distance, being lower for Rep. 4 than for Rep. 2, even though both are valid. This can be due to the fact that the wind
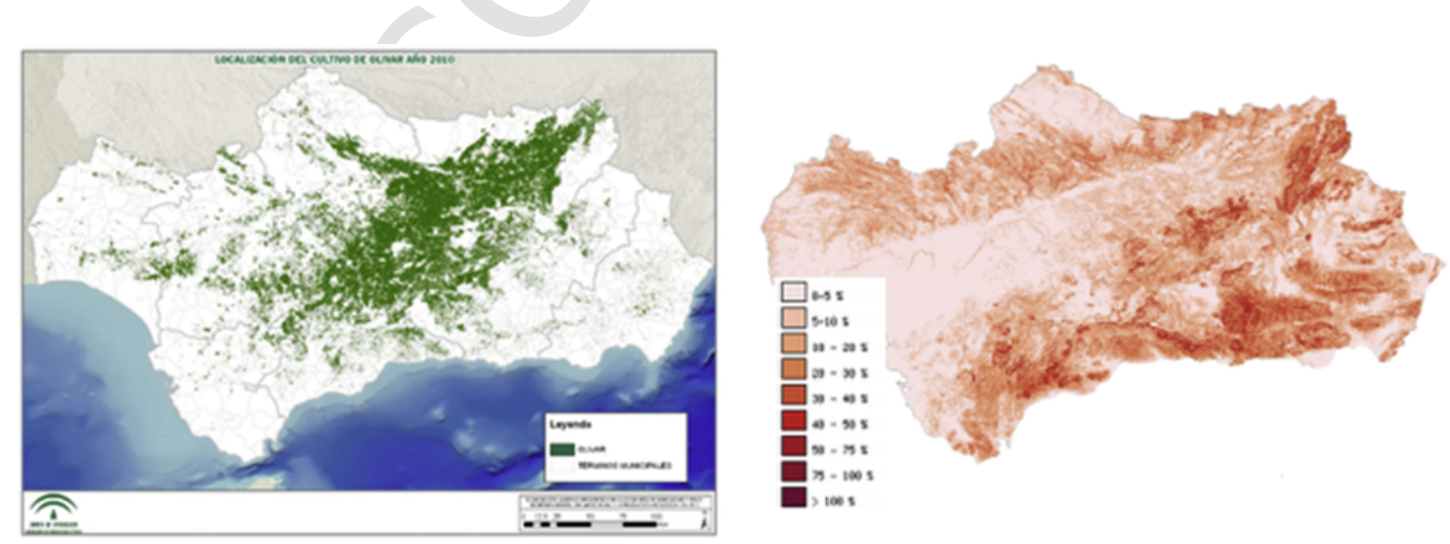

Fig. 6. Left: localization of olive fields in Andalucía area (Junta de Andalucía, 2015b). The maps show the mean slope by the town (Junta de Andalucía, 2015c). 
Table 3

Summary of the canopy characteristics.

\begin{tabular}{llllll}
\hline Nomenclature & Definition and units & Mean & Min & Max & $\begin{array}{l}\text { Standard } \\
\text { deviation }\end{array}$ \\
\hline $\mathrm{H}_{\mathrm{FL}}$ & Height first leaf $(\mathrm{m})$ & 0,56 & 0,30 & 0,80 & 0,04 \\
$\mathrm{H}_{\mathrm{T}}$ & Total height $(\mathrm{m})$ & 4,66 & 4,10 & 5,00 & 0,06 \\
$\mathrm{MV}$ & Mean vector $(\mathrm{m})$ & 3,08 & 2,46 & 3,69 & 0,10 \\
$\mathrm{E}_{\mathrm{V}}$ & Ellipsoid volume & 80,26 & 41,11 & 131,27 & 6,06 \\
& $\left(\mathrm{~m}^{3}\right)$ & & & & \\
$\mathrm{A}_{\mathrm{PA}}$ & Projected area $\left(\mathrm{m}^{2}\right)$ & 27,48 & 17,61 & 39,23 & 1,75 \\
$\mathrm{~V}_{\mathrm{L}}$ & LiDAR volume $\left(\mathrm{m}^{3}\right)$ & 61,88 & 27,47 & 110,72 & 6,25 \\
\hline
\end{tabular}

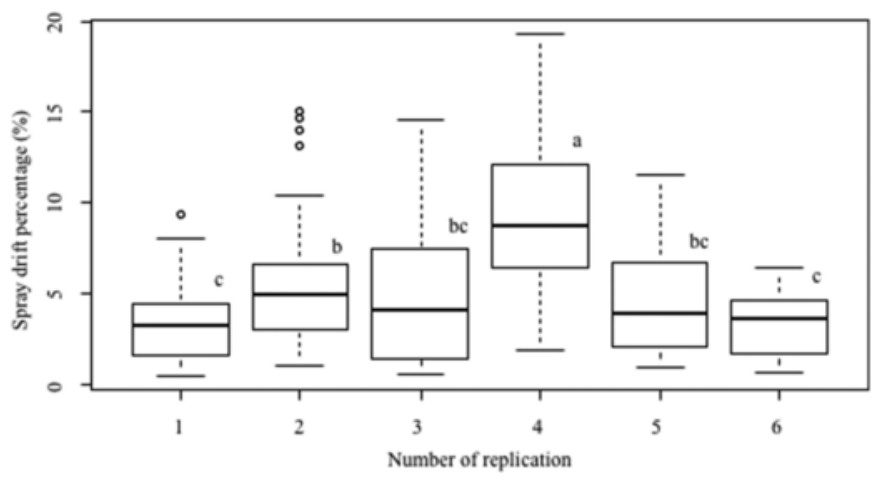

Fig. 7. Box-plot graphic of horizontal deposition samples for each of the six replications of the trial. Same letter means the means are not significantly different.

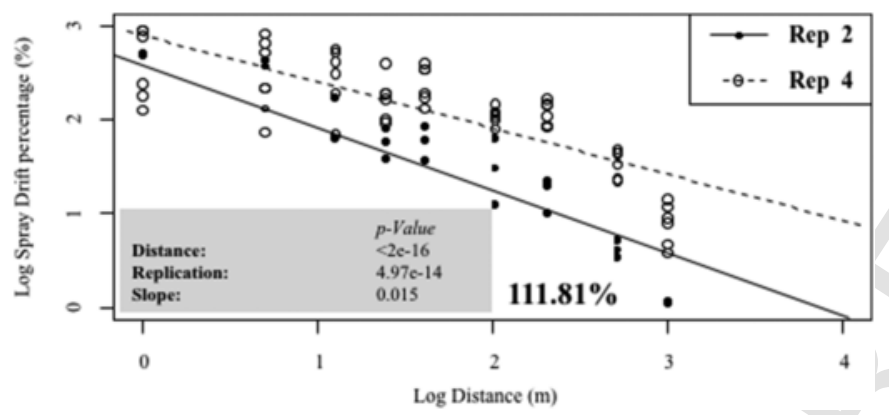

Fig. 8. ANCOVA between replications 2 and 4 . The red values indicate the percentage difference between the replications (Replication 2 vs. 4). (For interpretation of the references to colour in this figure legend, the reader is referred to the web version of this article.)

speed in Rep. 4 was almost double than in Rep. $2,2.3 \mathrm{~m} \cdot \mathrm{s}^{-1} \mathrm{vs}$ $1.4 \mathrm{~m} \cdot \mathrm{s}^{-1}$ respectively, what could have produced a longer drag of drop cloud and higher deposits at higher distances. Regarding the percentage difference between the collected deposits, the difference of $11.81 \%$ is noticeable but not high.

Fig. 10 shows the multiple comparisons of Reps. 2 and 4 with each of the non-valid replications. As expected, the co-variable distance had a significant effect in all cases, with $P$-values of $<2 \cdot 10^{-16}$. Regarding the replication factor, there are significant differences in all cases, with P-values that vary but which are all lower than 0.05 . In the case of Rep. 2, for the slope factor, there were significant differences only between it and Rep. 1, for which the P-value is around 0.05 (0.047). This fact indicates a similar trend in the collected deposit for a given distance, even when the other replications were considered invalid given that they did not satisfy the requirements of the norm. For the Rep. 4 comparisons, there were significant differences in the slope for all cases except for Rep. 6, but with a P-value of 0.058. As can be seen, the differences between Rep. 2 and Reps. 1, 3, 5 , and 6 are greatly reduced, with values of $11.1 \%, 0.9 \%, 2.4 \%$, and
$20.6 \%$, respectively. On the other hand, the differences between Reps. 4 and $1,3,5$ and 6 are much more noticeable, with values of $20.5 \%$, $11.4 \%, 12.7 \%$, and $29.1 \%$.

A very controversial aspect of the setup of the trials is the placement of the ground collectors in relation to the position of the trees. Fig. 11 shows the drift deposit patterns for the horizontal collectors placed either between or behind the trees, according to the scheme shown in Fig. 1.

Fig. 12 summarises the vertical distribution of the spray deposits collected from the nylon collectors hung from the vertical masts. The data exhibits a similar response for the different heights, with a decreasing trend with an increase in the height. It should be noted that the high values obtained at the highest sampling points are equal to approximately $75 \%$ of those obtained at the lowest sampling points. Nevertheless, the difference between Reps. 2 and 4 are very marked, with the second being almost double the first for every height. Furthermore, as shown by the ground deposits, Rep. 2 is much more similar to the other replications than Rep. 4, with this last deposit value being much higher than those for all the others.

\subsection{Proposed drift curve for traditional olive trees plantations}

Considering the results obtained in the ground collectors, Fig. 13 shows the proposed drift curve for traditional olive trees plantations. It considers the results of all replicates except of replicate 4 since, as it is showed before, it follows the ISO requirement but has a very different tendency that the rest. Following the established procedure (Rautmann et al., 2001), coefficients of the exponential function to define the proposed olive drift curve are shown in Table 5 distinguishing two different ranges: distance $<15 \mathrm{~m}$; and distance $>15 \mathrm{~m}$, following Rautmann et al. (2001).

The proposed drift curve for traditional olive trees shows the same tendency that the ones for orchards and hops. However, it is clearly detected an important reduction of drift values in the zone close to the edge row of the parcel $(0-10 \mathrm{~m})$ in both cases. This fact can be explained by the differences in canopy structure and vigour of the trees, with traditional olive trees denser and with a larger structure than those used for the establishment of the actual drift curves. For the distances over $10 \mathrm{~m}$, in general the drift curve for olive trees results on a lower drift values than those from the actual standards; however, the differences are much less remarkable when it is compared with the fruit crop drift curve at spray drift percentage. On the other side, the drift curve for hops is clearly different that the one proposed for traditional olive trees for spray drift percentage. Fig. 14 shows the statistical comparison of this curve with the standard drift curves for fruit crop late growth stage (upper part of the figure) and hops (bottom part of the figure) after applying the ANCOVA test.

\section{Discussion}

The executed field trials allowed to obtain a representative series of drift curves in traditional olive trees plantations, which has been used to develop a reference drift curve for this type of plantations. Results allows to propose a most close reference curve for drift evaluation in this important agricultural sector, considering the special canopy characteristics, far away from that representing fruit orchard trees or hops.

The aforementioned problems related with the field selection process represented an important issue to be solved prior to start the field trials. This problem arises from trying to perform a test under real-world conditions, due to the lack of adaptation of the requirements to this particular crop. The most limiting factor was deter- 

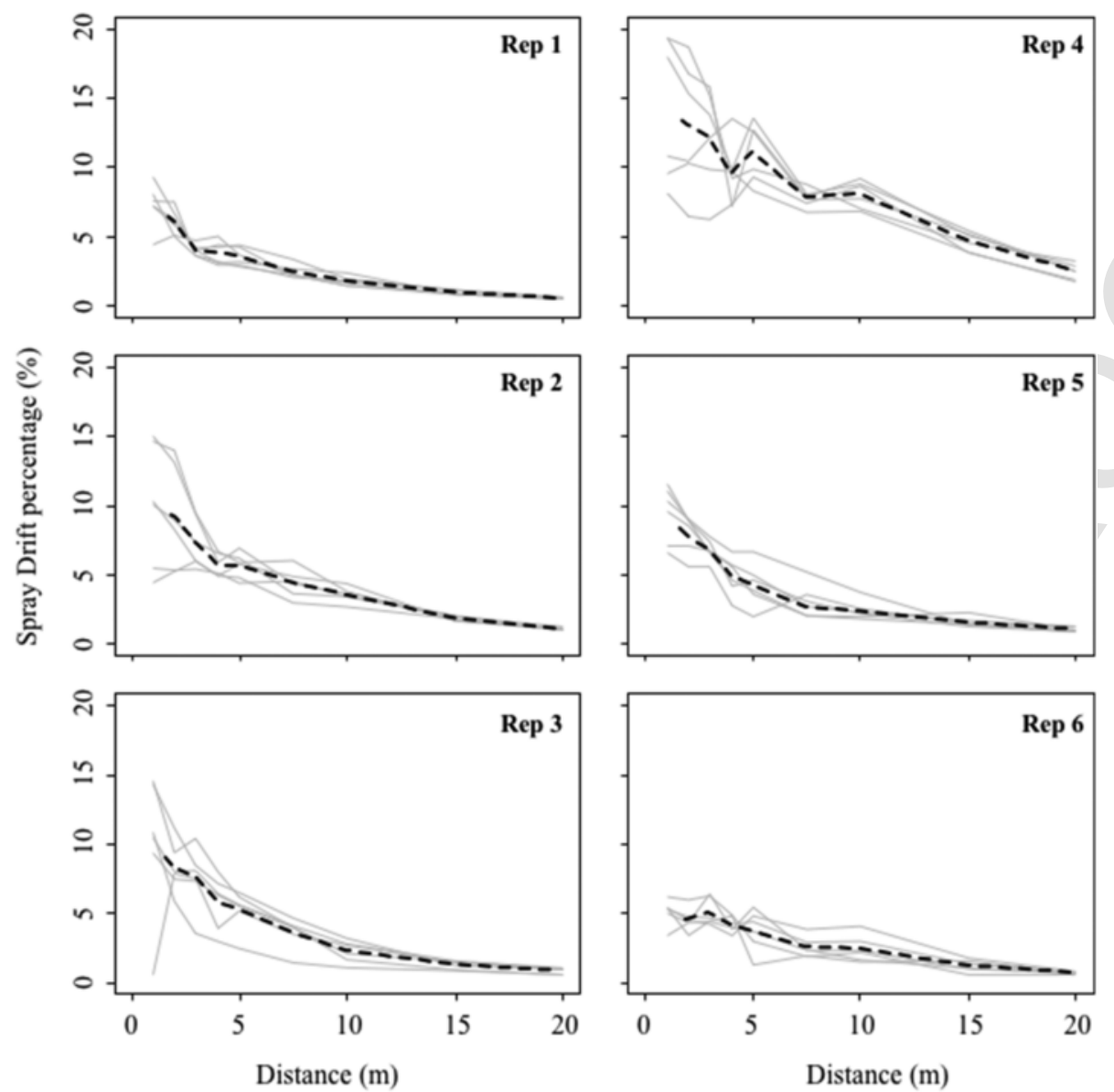

Fig. 9. Drift curve distribution at each position (A to F, grey lines) for each replication. The dotted lines represent the mean curves.

Table 4

Transformed equations and P-values for each one of the 6 replications of the trials.

\begin{tabular}{lllll}
\hline Repetition & Logarithm function & Adjusted R-Squared & $P$ value & Significance \\
\hline Rep 1 & $\mathrm{Y}=2.29 * \mathrm{X}-0.8$ & 0.87 & $<2.2 \mathrm{e}-16$ & $* * *$ \\
Rep 2 & $\mathrm{Y}=2.58 * \mathrm{X}-0.67$ & 0.75 & $<2.2 \mathrm{e}-16$ & $* * *$ \\
Rep 3 & $\mathrm{Y}=2.56 * \mathrm{X}-0.79$ & 0.60 & $2.218 \mathrm{e}-12$ & $* * *$ \\
Rep 4 & $\mathrm{Y}=2.89 * \mathrm{X}-0.49$ & 0.61 & $1.364 \mathrm{e}-12$ & $* * *$ \\
Rep 5 & $\mathrm{Y}=2.52 * \mathrm{X}-0.75$ & 0.85 & $<2.2 \mathrm{e}-16$ & $* * *$ \\
Rep 6 & $\mathrm{Y}=2.05 * \mathrm{X}-0.68$ & 0.68 & $6.449 \mathrm{e}-15$ & $* * *$ \\
\hline
\end{tabular}

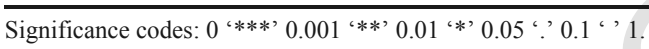

mined to be the need for bare space next to the site. In the most suitable cases, in which there were arable fields next to the olive groves, there was usually some kind of physical limitation that made impossible to set up the trial. The regularity of the field border, its placement as much perpendicular as possible to the tree rows, and the irregularities in the plantation patterns were other important factors that had to be taken into account.

Regarding the slope, it was proved almost impossible to find horizontal surfaces next to the olive groves, seriously limiting the use of the vertical collectors. In addition, the ground deposits would not be comparable to those obtained for other crops due to the positions not being the same, even though the same sampling points are used. The overall result of the process was one entire month dedicated to site selection, with the ultimate result being that only one field could be used from among all those selected for study. Furthermore, it was im- possible to ensure that the environmental conditions will conform to the general trends during the trial.

The current standard for drift measurement (ISO 22866) includes very restrictive requirements for the suitability of the environmental conditions, especially in terms of wind speed and direction.

Considering the canopy characteristics, the tree dimensions and the plantation pattern have an important effect on the complete application of the actual standard for drift measurements. In the first instance, the 50-m distance from the tractor takes in fewer than five trees, so the placement of the collectors represents a very critical point and, as it was stated in the Results section, it has a great bearing on the trial outcome. The irregularity of the crown shapes, as shown by the results of the canopy characterisation, could also be of great importance, making it difficult to replicate the trial in different locations, even at the same site.

Focusing on the distribution of the spray drift on the ground collectors, the first noticeable aspect is the significant difference among all the replications, which exhibited a very low repeatability in the trial.

Wind speed values during replicate. 2 was different from that of the other replications. This aspect significantly affected the drift amount in previous studies (Arvidsson et al., 2011) and in Rep. 2 was approximately double that in the other cases, as can be seen in Table 2. The wind direction, on the other hand, is more irregular, so its effect seems to be less important than the wind speed. If this is the case, more flexible limits could be set for the wind direction or for its regu- 

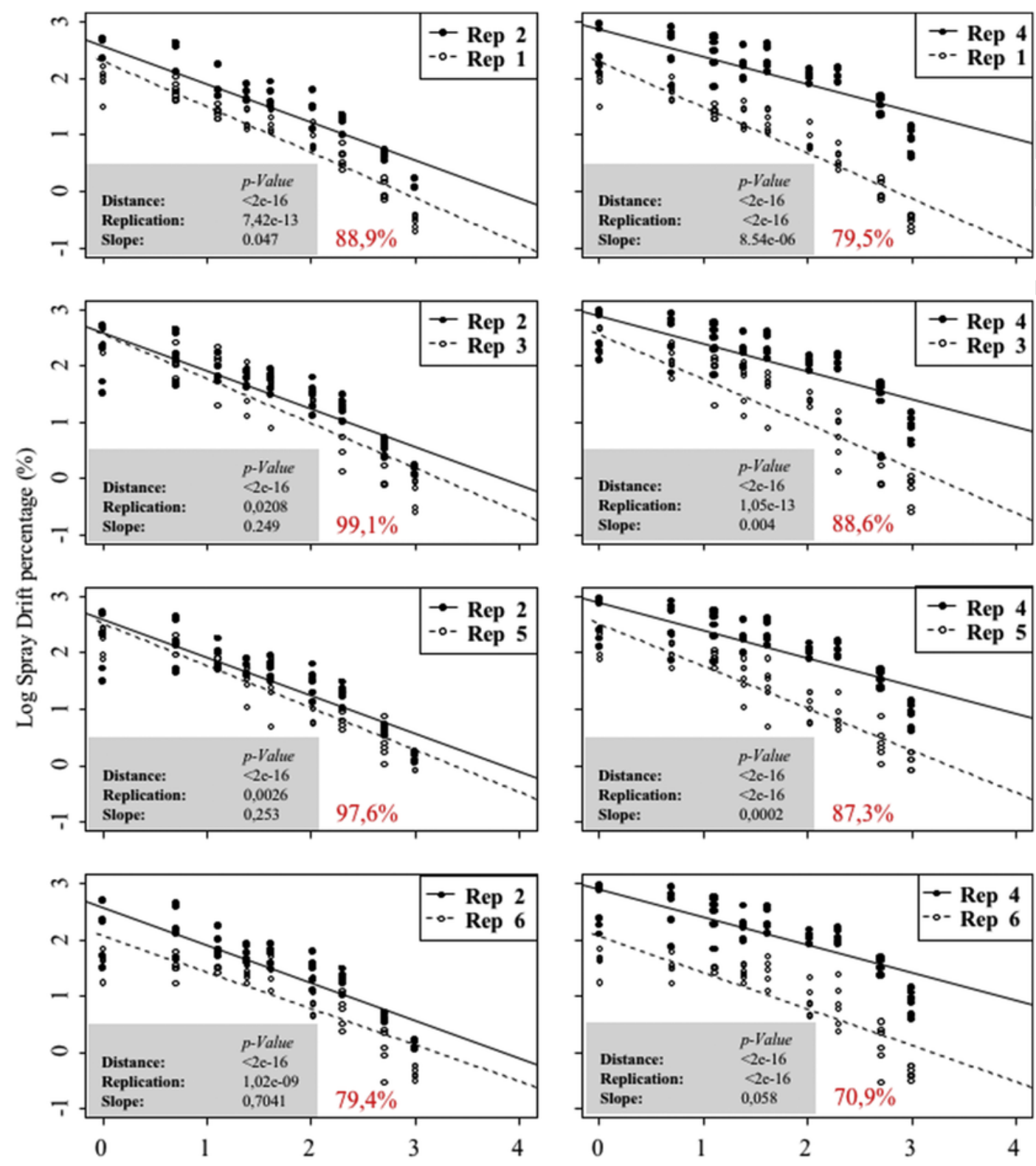

Log Distance $(\mathrm{m})$
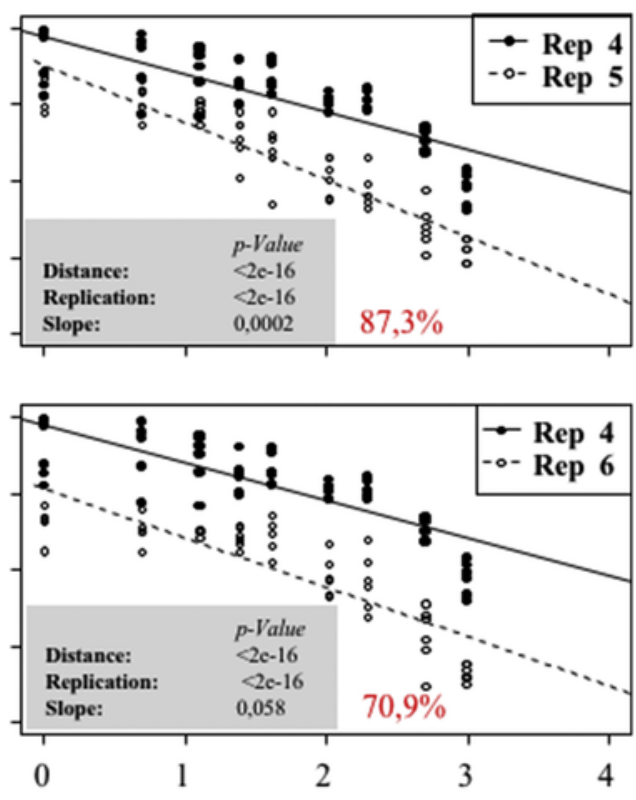

Log Distance $(\mathrm{m})$

Fig. 10. ANCOVA results for multiple comparisons between the replications that were accepted $(2$ and 4$)$ and not accepted $(1,3,5$, and 6$)$.

larity. For that reason, Rep. 2 was not used to develop the proposed drift curve for traditional olive trees plantations.

Considering airborne drift results, represented by the absolute value of the tracer deposit measured in the airborne collectors, their trends are very similar in every replication. It is important to state that the limitation that states that $<10 \%$ should be collected in the last sample is difficult to satisfy. The height of the olive trees makes it necessary for, in order to reach the upper levels of the canopy, a high proportion of the pesticide to be applied to those. Therefore, drift masts should be higher than $6 \mathrm{~m}$ to capture the entire drift cloud, such that there is only a small percentage captured by the upper sampling pieces. Furthermore, the information given by them seems to be very similar to that of the ground collector, but less complete and, in addition, the high mean slopes present in the groves makes their use diffi- cult, as was mentioned previously. Because of all these issues, their application to this crop would appear to be difficult.

\section{Conclusions}

A field tests series was carried out in order to evaluate the adaptability of the current standard ISO 22866 for drift measurement in traditional olive grove. The following conclusions can be drawn:

- A new proposed drift curve for traditional olive trees plantations has been developed, and important differences have been underlined when a deep comparison with the actual standard curves have been obtained.

- ISO 22866 can be adapted to olive groves but there are major difficulties affecting the trial protocol and the accurate site selection, 

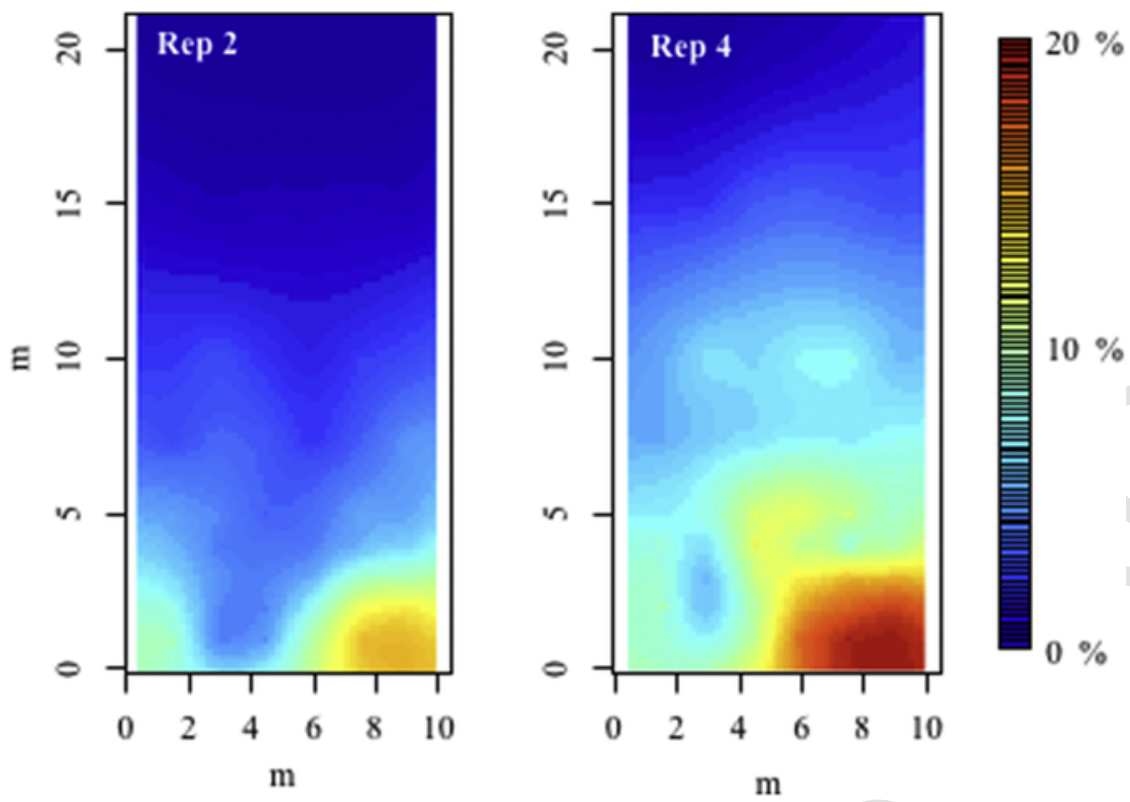

Fig. 11. Percentage spray drift map.

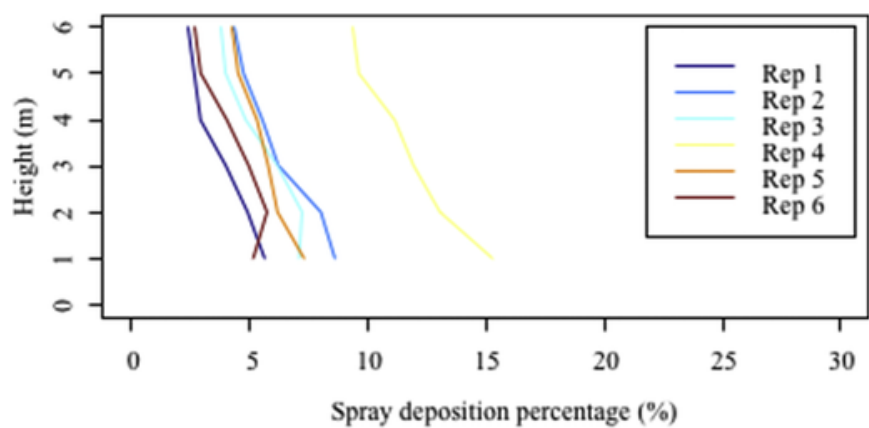

Fig. 12. Mean deposits collected on all nylon collectors at each height on vertical masts. Values represent the average deposition measured in the two masts placed at $5 \mathrm{~m}$ and $10 \mathrm{~m}$ from the sprayer track.

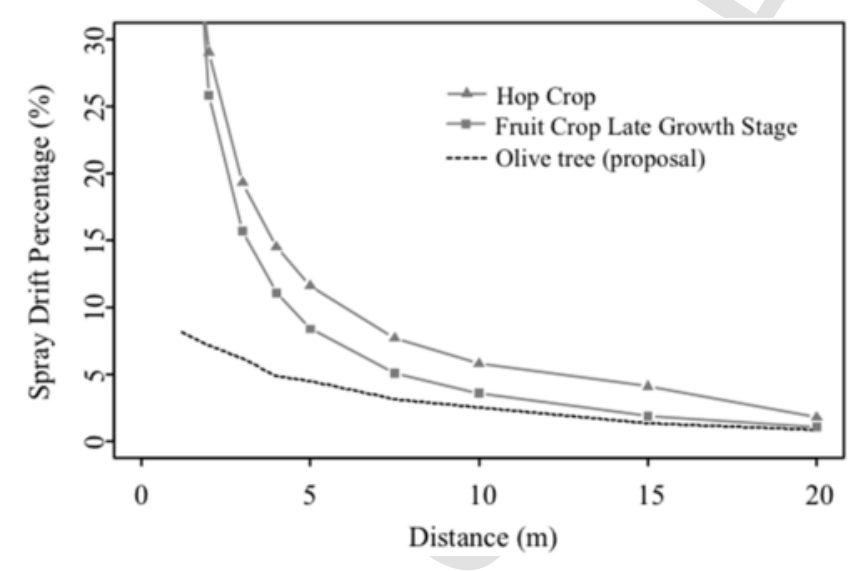

Fig. 13. Proposal of the drift curve for traditional olive trees plantations and its comparison with the actual drift curves for fruit crop late growth stage and hops proposed by Rautmann et al. (2001).
Table 5

Parameters of the exponential function for drift curves $\left(y=a * x^{b}\right)$.

\begin{tabular}{llll}
\hline Crop & Distance (X value) & $\mathrm{a}$ & $\mathrm{b}$ \\
\hline Fruit crop late stage $^{\mathrm{a}}$ & $<15 \mathrm{~m}$ & 60.36 & $-1,2243$ \\
& $\geq 15 \mathrm{~m}$ & 298.83 & 1.8672 \\
Hops $^{\mathrm{a}}$ & $<15 \mathrm{~m}$ & 58.271 & 1.0043 \\
& $\geq 15 \mathrm{~m}$ & 9205.3 & 2.8527 \\
Olive trees traditional plantation & $<15 \mathrm{~m}$ & 11.061 & -0.734 \\
& $\geq 15 \mathrm{~m}$ & 93.637 & -1.575 \\
\hline
\end{tabular}

a Proposed in Rautmann et al. (2001).

mainly due to the specific characteristics of the canopy and layout of such particular crop.

- The very restrictive requirements of the actual standard make it very difficult to obtain valid replications of the trials, even after dedicating considerable time and effort.

- The use of vertical collectors represents a very difficult task due to the high slopes usually present in olive orchards.

- The repeatability of the trials was proved to be very low, with important differences arising between the valid replications of the same test. The replication factor significantly affected all of the comparisons between the replications.

As a general conclusion, it can be suggested that the application of actual ISO 22866 and the adoption of the actual standard curves needs to be updated considering the specific characteristics of plantations, as it has seen for the case of traditional olive trees.

\section{Uncited references}

\section{Acknowledgements}

The authors thank the Ministry of Economy and Competitiveness of the Spanish government for their economic support through the pre-commercial procurement Mecaolivar project, financed with FEDER funds, and the AgVANCE project (AGL2013-48297-C2-1-R). The first author acknowledges the support of the Spanish Ministry of Education, Culture and Sport for its financial support through 

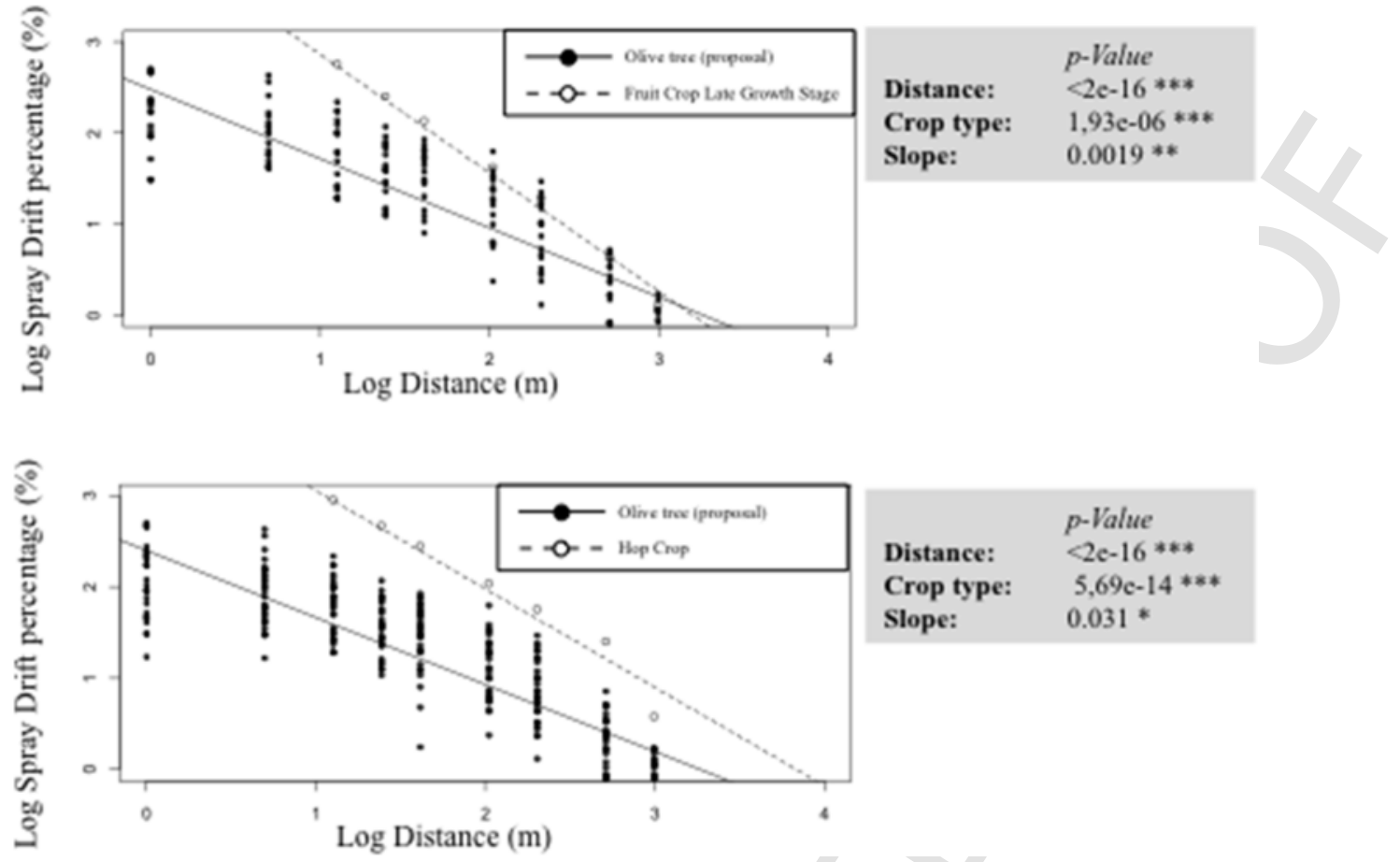

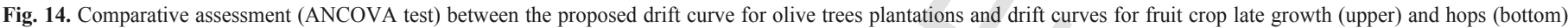
proposed by Rautmann et al. (2001)

the National Training Program of University Lecturers (FPU). We also appreciate the support of the Spanish olive oil inter-trade (IAOE).

\section{References}

Arvidsson, T., Bergström, L., Kreuger, J., 2011. Spray drift as influenced by meteorological and technical factors. Pest Manag. Sci. 67, 586-598. https://doi.org/10. $1002 /$ ps.2114

Balsari, P., Marucco, P., Tamagnone, M., 2005. Technical note: a system to assess the mass balance of spray applied to tree crops. Trans. ASAE 48, 1689-1694.

Balsari, P., Marucco, P., Tamagnone, M., 2007. A test bench for the classification of boom sprayers according to drift risk. Crop Prot. 26, 1482-1489. https://doi.org/ 10.1016/j.cropro.2006.12.012.

Balsari, P., Gil, E., Marucco, P., van de Zande, J.C., Nuyttens, D., Herbst, A., Gallart, M., 2017. Field-crop-sprayer potential drift measured using test bench: effects of boom height and nozzle type. Biosyst. Eng. 154, 3-13. https://doi.org/10.1016/j. biosystemseng.2016.10.015.

Burn, A., 2003. Pesticide buffer zones for the protection of wildlife. Pest Manag. Sci. 59, 583-590. https://doi.org/10.1002/ps.698.

Core Team, R., 2012. R: a language and environment for statistical computing. In: R Foundation for Statistical Computing, Retrieved from www.r-project.org.

De Schampheleire, M., Baetens, K., Nuyttens, D., Spanoghe, P., 2008. Spray drift measurements to evaluate the Belgian drift mitigation measures in field crops. Crop Prot. 27, 577-589. https://doi.org/10.1016/j.cropro.2007.08.017.

Doruchowski, G., Roettele, M., Herbst, A., Balsari, P., 2013. Drift evaluation tool to raise awareness and support training on the sustainable use of pesticides by drift mitigation. Comput. Electron. Agric. 97, 27-34. https://doi.org/10.1016/j.compag. 2013.06.006

Doruchowski, G., Balsari, P., Gil, E., Marucco, P., Roettele, M., Wehmann, H.-J., 2014. Environmentally optimised sprayer (EOS) - a software application for comprehensive assessment of environmental safety features of sprayers. Sci. Total Environ. 482-483, 201-207.

FOCUS, 2001. FOCUS Surface Water Scenarios in the EU Evaluation Process Under 91/414/EEC.

FOCUS, 2014. FOrum for Co-ordination of pesticide fate models and their USe. Retrieved from http://focus.jrc.ec.europa.eu/.

Ganzelmeier, H., Rautmann, D., Spangenberg, R., Streloke, M., Herrmann, M., Wenzelburger, H.J., Walter, H.F., 1995. Studies of the spray drift of plant protection products. In: Results of a Test Program Carried Out Throughout the Federal Republic of Germany. Blackwell, Berlin.

García-Ramos, F.J., Malón, H., Aguirre, A.J., Boné, A., Puyuelo, J., Vidal, M., 2015. Validation of a CFD model by using 3D sonic anemometers to analyse the air velocity generated by an air-assisted sprayer equipped with two axial fans. Sensors (Switzerland) 15, 2399-2418. https://doi.org/10.3390/s150202399.

Gil, E., Llorens, J., Llop, J., Fàbregas, X., Gallart, M., 2013. Use of a terrestrial LIDAR sensor for drift detection in vineyard spraying. Sensors 13, 516-534. https:// doi.org/10.3390/s130100516.

Gil, E., Balsari, P., Gallart, M., Llorens, J., Marucco, P., Andersen, P.G., Fàbregas, X., Llop, J., 2014. Determination of drift potential of different flat fan nozzles on a boom sprayer using a test bench. Crop Prot. 56, 58 - 68 .

Girardeau-Montaut, D., 2011. CloudCompare, a 3D Point Cloud and Mesh Processing. Open Source Project. EDF Research and Development, Telecom ParisTech, Retrieved April 1, 2015, from http://danielgm.net/cc/.

Gregorio, E., Rocadenbosch, F., Sanz, R., Rosell-Polo, J., 2015. Eye-safe lidar system for pesticide spray drift measurement. Sensors 15, 3650-3670. https://doi.org/10. $3390 / \mathrm{s} 150203650$.

Grella, M., Marucco, P., Manzone, M., Gallart, M., Balsari, P., 2017. Effect of sprayer settings on spray drift during pesticide application in poplar plantations (Populus spp.). Sci. Total Environ. 578, 427-439.

Herbst, A., 2001. A Method to Determine Spray Drift Potential From Nozzles and its Link to Buffer Zone Restrictions. ASAE (Paper No. 01-1047).

ISO 22401, 2015. Equipment for Crop Protection - Method for Measurement of Potential Spray Drift From Horizontal Boom Sprayers by the Use of a Test Bench.

ISO 22856, 2008. International Standard. . Equipment for Crop Protection - Methods for the Laboratory Measurement of Spray Drift - Wind Tunnels.

ISO 22866, 2005. International Standard. Equipment for Crop Protection - Methods for Field Measurement of Spray Drift.

Junta de Andalucía, 2015a. El Olivar Andaluz (in Spanish). Available at: http://www. juntadeandalucia.es/agriculturaypesca/prospectiva/, Accessed on: 14th June 2015.

Junta de Andalucía, 2015b. El Sector Agrario y Pesquero en Andalucía (in Spanish). Available at: http://www.juntadeandalucia.es/agriculturaypesca/portal/ servicios/estadisticas/estudios-e-informes/el-sector-agrario-en-andalucia.html, Accessed on: 15th June 2015 .

Junta de Andalucía, 2015c. El Olivar Andaluz (in Spanish). Available at: http://www. juntadeandalucia.es/medioambiente/site/rediam/menuitem, Accessed on: 14th June 2015.

Llorens, J., Gil, E., Llop, J., Escolà, A., 2011. Ultrasonic and LIDAR sensors for electronic canopy characterization in vineyards: advances to improve pesticide application methods. Sensors 11, 2177-2194. https://doi.org/10.3390/s110202177. 
Miranda-Fuentes, A., Llorens, J., Gamarra-Diezma, J., Gil-Ribes, J., Gil, E., 2015. Towards an optimized method of olive tree crown volume measurement. Sensors 15 , 3671-3687. https://doi.org/10.3390/s150203671.

Ozkan, H.E., 1998. Effects of major variables on drift distances of spray droplets. In: Food, Agric. and Biol. Eng. Extension FactSheet AEX. pp. 525-598.

Parliament, E, 2009. Directive 2009/127/EC of the European Parliament and of the Council of 21 October 2009 Amending Directive 2006/42/EC with Regard to Machinery for Pesticide Application.

Parliament, E, 2009. Directive 2009/128/EC of the European Parliament and of the Council of 21 October 2009 Establishing a Framework for Community Action to Achieve the Sustainable Use of Pesticides.

Parliament, E, 2009. Regulation (EC) No 1107/2009 of the European Parliament and of the Council Concerning the Placing of Plant Protection Products on the Market and Repealing Council Directives 79/117/EEC and 91/414/EEC.

Pergher, G., 2001. Recovery rate of tracer dyes used for spray deposit assessment. Trans. ASABE 44, 787-794.

Poprawski, T., Carruthers, R., Speese, J., Vacek, D., Wendel, L., 1997. Early-season applications of the fungus Beauveria bassiana and introduction of the hemipteran predator Perillus bioculatus for control of Colorado potato beetle. Biol. Control 10, 48-57. https://doi.org/10.1006/bcon.1997.0537.

Rautmann, D., Streloke, M., Winkler, R., 2001. New basic drift values in the authorization procedure for plant protection products. Mitt. Biol. Bundesanst. Land Forstwirtsch 383, 133-141.

Ravier, I., Haouisee, E., Clément, M., Seux, R., Briand, O., 2005. Field experiments for the evaluation of pesticide spray-drift on arable crops. Pest Manag. Sci. 61, 728-736. https://doi.org/10.1002/ps.1049.

Rimmer, D., Johnson, P.D., Kelsey, A., Warren, N.D., 2009. Field experiments to assess approaches for spray drift incident investigation. Pest Manag. Sci. 65, 665-671. https://doi.org/10.1002/ps.1734.
Serrano, L., Filella, I., Peñuelas, J., 2000. Remote sensing of biomass and yield of winter wheat under different nitrogen supplies. Crop Sci. 40, 723. https://doi.org/10. 2135/cropsci2000.403723x

de Snoo, G.R., de Wit, P.J., 1998. Buffer zones for reducing pesticide drift to ditches and risks to aquatic organisms. Ecotoxicol. Environ. Saf. 41, 112-118. https://doi. org/10.1006/eesa.1998.1678.

Tavares, R.M., Cunha, J.P.A.R., Alves, T.C., Bueno, M.R., Silva, S.M., Zandonadi, C.H.S., 2017. Electrostatic spraying in the chemical control of Triozoida limbata (Enderlein) (Hemiptera: Triozidae) in guava trees (Psidium guajava L.). Pest Manag. Sci. 73, 1148-1153. https://doi.org/10.1002/ps.4433.

Wang, M., Rautmann, D., 2008. A simple probabilistic estimation of spray drift factors determining spray drift and development of a model. Environ. Toxicol. Chem. 27 (12), 2617-2626. https://doi.org/10.1897/08-109.1.

Xu, W., Su, Z., Feng, Z., Xu, H., Jiao, Y., Yan, F., 2013. Comparison of conventional measurement and LiDAR-based measurement for crown structures. Comput. Electron. Agric. 98, 242-251. https://doi.org/10.1016/j.compag.2013.08.015.

van de Zande, J.C., Porskamp, H.A.J., Michielsen, J.M.G.P., Holterman, H.J., Huijsmans, J.F.M., 2000. Classification of spray applications for driftability, to protect surface water. Asp. Appl. Biol. 66, 57-65.

van de Zande, J.C., Holterman, H.J., Wenneker, M., 2008. Nozzle classification for drift reduction in orchard spraying; identification of drift Reduction Class threshold nozzles. In: Agric. Eng. Int. CIGR Ejournal X. pp. 1-12.

van de Zande, J.C., Wenneker, M., Michielsen, J.M.G.P., Stallinga, H., 2015. Spray drift and resident risk in orchard spraying; reference and spray drift reducing techniques. In: Suprofruit 2015. 13th Workshop on Spray Application Techniques in Fruit Growing. Lindau, Germany. https://doi.org/10.1371/journal.pone.0120725. 